\title{
ChemComm
}

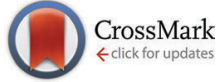

Cite this: Chem. Commun., 2014, 50,15341

Received 3rd September 2014, Accepted 10th October 2014

DOI: $10.1039 /$ c4cc06943a

www.rsc.org/chemcomm

\section{Directed one-pot syntheses of crown ether wheel-containing main chain-type polyrotaxanes with controlled rotaxanation ratios $\dagger$}

\author{
Kazuko Nakazono, Tomonori Ishino, Tomoyuki Takashima, Daisaku Saeki, \\ Daisuke Natsui, Nobuhiro Kihara and Toshikazu Takata*
}

\begin{abstract}
The directed synthesis of main chain-type polyrotaxanes possessing crown ether wheels was successfully achieved through two methods, A and B. Method A involved the direct wheel threading of poly(secammonium salt) followed by end-capping with a bulky group, while method B utilized polyaddition of a pseudo[2]rotaxane monomer to facilitate the control of the structure, i.e. the rotaxanation ratio.
\end{abstract}

The first synthesis of a polypseudorotaxane (PPRX) from cyclodextrin (CD) and a linear polymer by Ogata $^{1}$ and its end-capping to give a polyrotaxane (CD-PRX) by Harada ${ }^{2}$ heralded significant progress in the science of main chain-type CD-PRX, opening the door to various applications ${ }^{3}$ such as cross-linked gels. ${ }^{4}$ Efficient syntheses of CD-PRX have now led to its actual use. ${ }^{5}$ However, little is known about PRXs having other wheel components, ${ }^{6,7}$ in particular crown ether (CE) wheels, despite Gibson's pioneering work on PRXs. ${ }^{8}$ Therefore, the synthesis of PRX (not poly[2]rotaxane ${ }^{9}$ ) with a sufficiently high or controlled CE content and effective end-cap groups is an important challenge in this area. ${ }^{8 a, 10} \mathrm{CE}$-ammonium-salt-type rotaxane chemistry has enabled the synthesis of end-capped $[5]^{6 d}$ and [20] rotaxanes; ${ }^{6, b}$ however, both rotaxanes exhibit low solubility because of their oligoionic structures. Since CE-ammonium interaction-driven self-assembly often achieves accurate supramolecular systems, the greater ease with which functional groups can be introduced into $\mathrm{CE}$ as compared to $\mathrm{CD}$, as has been proven in rotaxane chemistry, ${ }^{11}$ strongly indicates the potential utility of CE-based main chain-type PRX (CE-PRX), and hence, its synthesis becomes of paramount importance. We have long studied the development of an effective synthetic method for CE-PRX with a controlled structure, and have found effective synthetic methods. ${ }^{12}$ A recent Grubbs' simple approach to CE-PRX via the polymerization of a CE-based pseudo[2]rotaxane monomer, ${ }^{13}$ has prompted us to report our results on the successful

Department of Organic and Polymeric Materials, Tokyo Institute of Technology,

2-12-1, O-okayama, Meguro-ku, Tokyo 152-8552, Japan.

E-mail: ttakata@polymer.titech.ac.jp

$\dagger$ Electronic supplementary information (ESI) available: Experimental procedures, spectra, SEC profiles, and DSC profiles. See DOI: 10.1039/c4cc06943a synthesis of CE-PRX with controlled structures. We report herein the directed one-pot synthesis of CE-PRXs by two reliable synthetic protocols to yield structure-definite PRXs with controlled number of wheel components and effective end-cap groups (Fig. 1), building on our recent results on the synthesis of dibenzo-24-crown-8ether (DB24C8)-containing PRXs. ${ }^{14}$ We also describe the simple modification to nonionic PRX that enables size-exclusionchromatographic (SEC) measurements.

Fig. 1 shows the two strategies for the synthesis of CE-PRXs. Method A consists of the initial wheel threading of poly(secammonium salt) and subsequent end-cap with a bulky agent. In the method $\mathrm{B}$, copolymerization of pseudo[2]rotaxane monomer and appropriate comonomer to CE-PRX by the successive end-cap to facilitate the control of the structure, i.e. the rotaxanation ratio.

Method A: direct wheel threading of poly(sec-ammonium salt) followed by end-capping. We started with this synthetic protocol because rotaxane synthesis by this route is usually known to proceed efficiently in one-pot, ${ }^{15}$ and it seems to be suitable for giving high-molecular-weight PRX. Although poly(sec-ammonium salt)

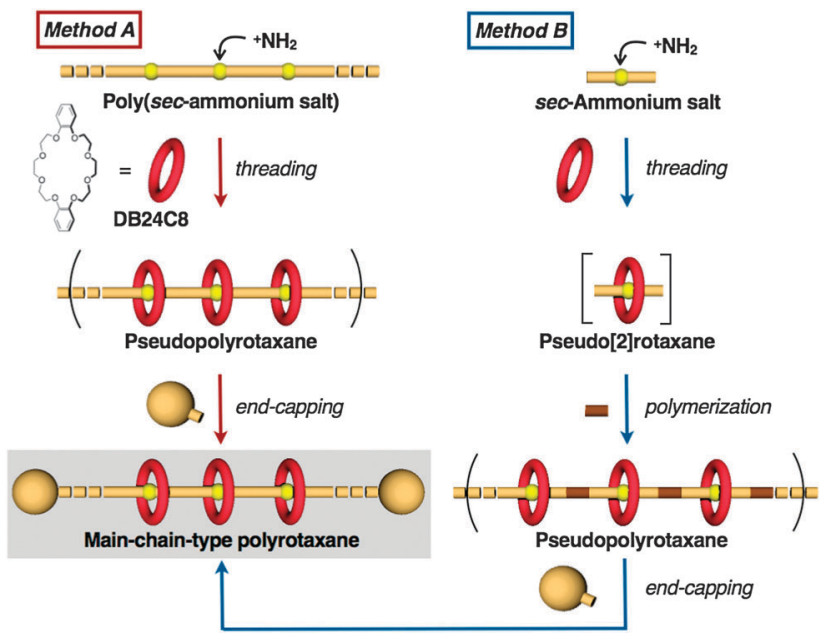

Fig. 1 Two independent strategies for preparing CE-based main chaintype PRXs via one-pot reaction. 
and the corresponding CE-PRX seemed insoluble in ordinary organic solvents because of their polyionic structures, we expected to observe CE-PRX formation because the oligorotaxane is more soluble than its axle polymer precursor oligo(sec-ammonium salt). ${ }^{6 a, b}$ We chose poly(hexamethyleneammonium $\mathrm{PF}_{6}$ ) (PHMAP) as the axle polymer because the hexamethylene spacer distance between the ammonium groups is appropriate for a high-yield synthesis of [3] rotaxane. ${ }^{6 e}$ PHMAP was obtained by the reduction of 6,6-nylon with $\mathrm{BH}_{3}$-THF complex ${ }^{16}$ followed by treatment with $\mathrm{NH}_{4} \mathrm{PF}_{6}$. PHMAP was relatively insoluble in organic solvents and the corresponding PPRX was obtained in only a minimal yield with DB24C8 in a $\mathrm{CHCl}_{3}-\mathrm{CH}_{3} \mathrm{CN}$ mixed solvent system. To address this problem, benzyl ammonium salt moieties were introduced at both PHMAP termini to enhance complexation capability. ${ }^{15,17}$ 4-Hydroxymethylbenzyl-terminated PHMAP 1 was prepared by condensation of amine-terminated 6,6-nylon and methyl 4-formylbenzoate followed by reduction and subsequent treatment with $\mathrm{NH}_{4} \mathrm{PF}_{6}$. The molecular weight of 1 was calculated by ${ }^{1} \mathrm{H}$ NMR to be $M_{\mathrm{n}}=3200$. One-pot synthesis of PRX 3 via intermediate PPRX 2 was carried out as follows (Scheme 1): a heterogeneous mixture of 1 and DB24C8 was allowed to stand and then 3,5-dimethylphenylisocyanate and a catalytic amount of dibutyltindilaurate (DBTDL) in solvent at room temperature was added to $\mathrm{it}^{15}$ (Table 1). The structure of PRX 3 was confirmed by spectroscopic analyses.

The reactions proceeded under heterogeneous conditions even with polar solvents such as $\mathrm{CH}_{3} \mathrm{CN}$ and $\mathrm{CH}_{3} \mathrm{NO}_{2}$ (Table 1). For PRX 3, the yield increased to $76 \%$ with increasing solvent polarity and hence the solubility of PPRX 2 during the endcapping process. For $\mathbf{1}$, the rotaxanation ratio (RR) of the $\mathrm{NH}_{2}$ group was sufficiently high $(>92 \%)$ without solvent dependency. These results suggest the following important reaction features: (i) the formation of PPRX 2 is accelerated by introducing a more strongly interactive unit (benzyl group) with CE at the axle termini; ${ }^{18}$ (ii) complexation between 1 and CE proceeds under heterogeneous conditions; and (iii) the CE wheel can

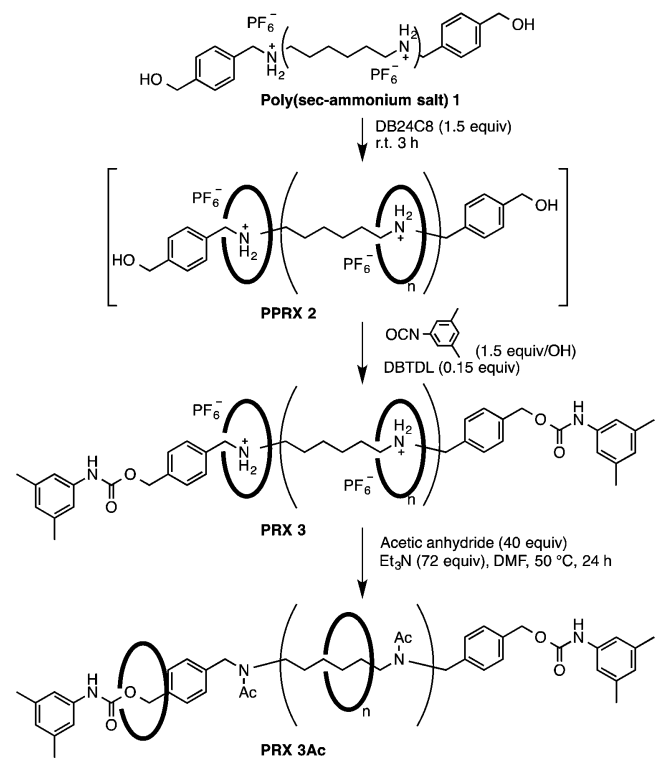

Scheme 1 Synthesis of PRX 3 and PRX $3 A c(0 \leq n \leq 1)$.
Table 1 Synthesis of PRX 3 by method A

\begin{tabular}{llll}
\hline Entry & Solvent & Yield of PRX $3^{a}(\%)$ & $\mathrm{RR}^{b}(\%)$ \\
\hline 1 & $\mathrm{CHCl}_{3}$ & 33 & 90 \\
2 & $\mathrm{ClCH}_{2} \mathrm{CH}_{2} \mathrm{Cl}$ & 58 & 98 \\
3 & $\mathrm{CH}_{3} \mathrm{CN}$ & 68 & 92 \\
4 & $\mathrm{CH}_{3} \mathrm{NO}_{2}$ & 76 & 98
\end{tabular}

${ }^{a}$ Calculated on the basis of $1 .{ }^{b}$ Rotaxanation ratio determined by ${ }^{1} \mathrm{H}$ NMR (range of error $\pm 2 \%$ ).

enter into the center of the axle polymer chain. For PRX 3, neutralization by $\mathrm{N}$-acetylation, ${ }^{19}$ which is required for estimating $M_{\mathrm{n}}$, was incomplete, presumably because CE is densely packed because of its short CE-CE distance. ${ }^{6 c}$ To address this issue, we turned to method B.

Method B: step polymerization of a reactive pseudo[2]rotaxane monomer followed by end-capping. We designed this synthetic protocol because of the reported efficiency of end-capping pseudo[2]rotaxane to give [2] rotaxane ${ }^{15}$ in addition to the high solubility of the monomer. ${ }^{6,20}$

We chose a dibenzylammonium-type axle $4^{17}$ because of the enhanced stability of the corresponding pseudorotaxane monomer. One-pot synthesis of PRX 6 was carried out as follows (Scheme 2): a mixture of pseudo[2]rotaxane (derived in situ from 4 and DB24C8) and diphenylmethane diisocyanate was treated with DBTDL in $\mathrm{CH}_{2} \mathrm{Cl}_{2}$. The resulting PPRX 5 was treated successively with 3,5-dimethylphenylisocyanate and 3,5-dimethylphenol to give PRX 6 selectively in high yield. The RR value and $M_{\mathrm{n}}$ were determined by ${ }^{1} \mathrm{H}$ NMR (Table 2).

Inspection of Table 2 reveals that method $\mathrm{B}$ is versatile and gives the desired CE-based PRXs in high yield. The RR values were controllable by the DB24C8 feed ratio; for example, 0.5-2.0 equiv. of DB24C8 yielded PRX 6 with RR values in the range 55-95\%. The end-capped structure of PRX 6 was confirmed by a decomposition study of PRX 6 in DMSO- $d_{6}$, which indicated that no DB24C8 was liberated from PRX 6 at ambient temperature. ${ }^{21}$ The structure of PRX 6 was determined using NMR, IR, and SEC. The ${ }^{1} \mathrm{H}$ NMR spectra clearly indicate the

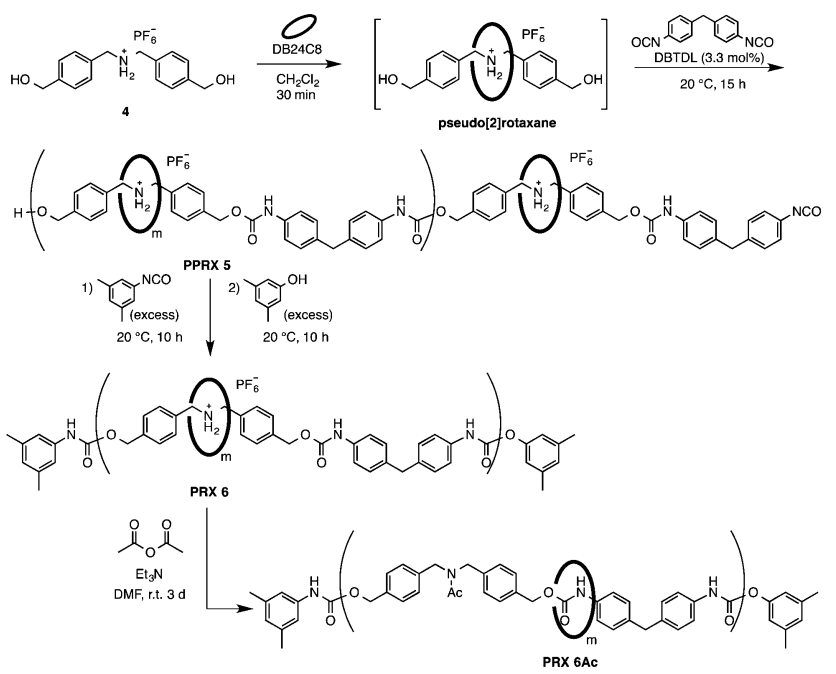

Scheme 2 Synthesis of PRX 6 and PRX 6Ac $(0 \leq m \leq 1)$. 
Table 2 Controlled synthesis of PRX 6 by method B ${ }^{a}$

\begin{tabular}{|c|c|c|c|c|c|c|c|}
\hline \multirow[b]{2}{*}{ Entry } & \multirow[b]{2}{*}{$\begin{array}{l}\mathrm{DB} 24 \mathrm{C} 8 / \mathrm{NH}_{2} \\
\text { (equiv.) }\end{array}$} & \multirow[b]{2}{*}{$\begin{array}{l}\text { Yield }^{b} \\
(\%)\end{array}$} & \multirow[b]{2}{*}{$\begin{array}{l}\mathrm{RR}^{c} \\
(\%)\end{array}$} & \multirow{2}{*}{$\begin{array}{l}\text { PRX 6 } \\
(\mathrm{NMR}) \\
M_{\mathrm{n}} / \\
10^{4} / \mathrm{Da}\end{array}$} & \multicolumn{3}{|c|}{ PRX 6Ac ${ }^{d}(\mathrm{SEC})$} \\
\hline & & & & & $\begin{array}{l}M_{\mathrm{n}} / \\
10^{4} / \mathrm{Da}\end{array}$ & $\begin{array}{l}M_{\mathrm{w}} / \\
10^{4} / \mathrm{Da}\end{array}$ & PDI \\
\hline 1 & 2.0 & 93 & 95 & 2.6 & 2.8 & 6.0 & 3.6 \\
\hline 2 & 1.0 & 84 & 90 & 2.5 & 4.2 & 9.3 & 2.2 \\
\hline 3 & 0.5 & 84 & 55 & 1.4 & 3.3 & 7.4 & 2.2 \\
\hline
\end{tabular}

${ }^{a} \mathbf{4}=0.5 \mathrm{M} .{ }^{b}$ Calculated on the basis of $4 .{ }^{c}$ Determined by ${ }^{1} \mathrm{H}$ NMR (range of error $\pm 2 \%$ ). ${ }^{d}$ Estimated by SEC (DMF, PSt) after N-acetylation.

involvement of the rotaxane structure, with DB24C8 localized at the ammonium moieties (Fig. 2). All signals are reasonably assignable; the two signals around 4.6 (A) and 4.8 (a) ppm indicate uncomplexed and complexed $N$-benzyl protons, respectively, whose ratio corresponds to the RR value.

To remove the polyionic nature from PRX 6, we subjected PRX 6 to N-acetylation with acetic anhydride and triethylamine. ${ }^{19}$ The acetylation reaction proceeded very efficiently to yield PRX 6Ac (93\% yield, Table 2, entry 1). Table 2 shows the SEC results of PRX 6Acs with different rotaxanation ratios (RR). All PRX 6Acs gave unimodal SEC profiles to support the occurrence of complete $\mathrm{N}$-acetylation of the ammonium moieties of PRX 6s, agreeing with other spectral data. ${ }^{22}$ The ${ }^{1} \mathrm{H}$ NMR spectrum of PRX 6Acs suggested two sets of signals assignable to the protons around the urethane moiety, although the axle component is symmetric. Thus, the conversion of the ammonium moiety caused the positional change of the crown ether wheel from the ammonium moiety to the urethane moiety probably due to the hydrogen bonding interaction as same as the model [2]rotaxane $\mathbf{S 9} .^{22}$ This is the first synthesis of a nonionic DB24C8-based main chain-type polyrotaxane. The solubility of the PRXs in typical organic solvents was investigated. PRX 3 was soluble in some polar solvents such as DMF and $\mathrm{CH}_{3} \mathrm{CN}$, whereas PRX 6 and PRX 6Ac showed the higher solubility to various solvents including acetone, as we expected. ${ }^{22}$

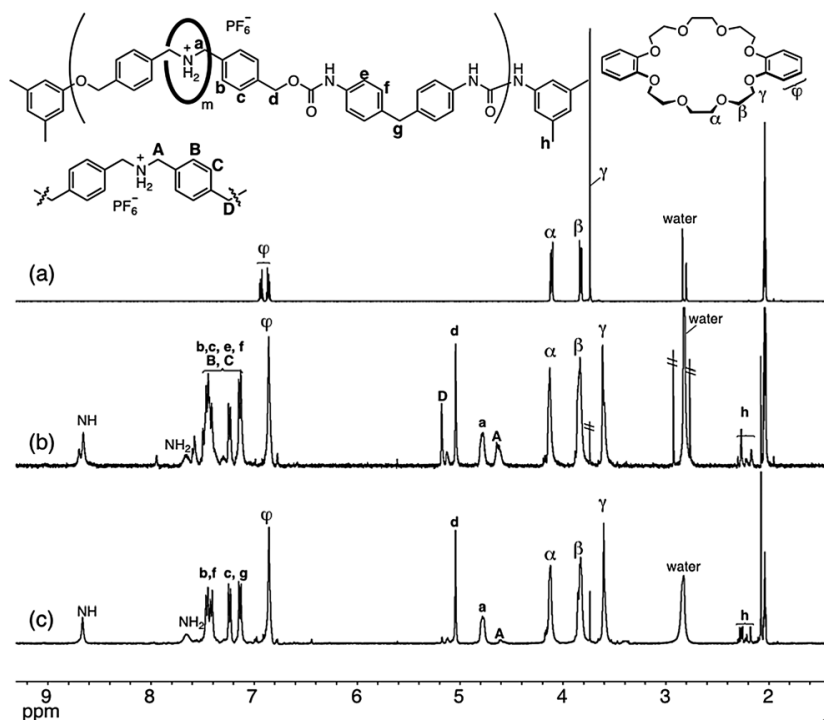

Fig. 2 Partial ${ }^{1} \mathrm{H}$ NMR spectra of (a) DB24C8, (b) PRX $6(\mathrm{RR}=55 \%)$, and (c) PRX $6(\mathrm{RR}=95 \%)\left(400 \mathrm{MHz},\left(\mathrm{CD}_{3}\right)_{2} \mathrm{CO}\right)$.

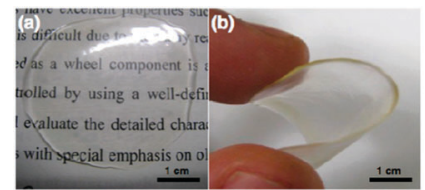

Fig. 3 Photographs of a transparent thin film of PRX $6(R R=95 \%)$ prepared by casting from the acetone solution.

In addition, the high molecular weight of PRX 6 was confirmed by its good film-forming property, which resulted in the formation of flexible transparent films by casting from the acetone solutions (Fig. 3). ${ }^{22}$

In conclusion, we have successfully accomplished the directed one-pot synthesis of a challenging target polymer in the PRX family: CE-based main chain-type PRXs. We showed that CE-PRXs are unusually soluble in typical organic solvents such as DMF, despite their polyionic structures. Method B, involving step polymerization of pseudo[2]rotaxane monomers, enables the controlled synthesis of PRXs possessing the desired rotaxanation ratios (RR values). Complete neutralization by the $\mathrm{N}$-acetylation of the ammonium moieties that promotes the $\mathrm{CE}$ translation on the axle ${ }^{23}$ proceeded remarkably well. A variety of modifications of CEs established so far enable versatile functionalization of PRXs. Thus, the present study heralds continuing rapid progress in the synthesis and application of CE-PRXs.

This work was financially supported by a Grant-in-Aid for Scientific Research from MEXT, Japan (No. 18064008 and 23245031), and K. N. thanks the Global COE program (Education and Research Center for Material Innovation) for the support.

\section{Notes and references}

1 N. Ogata, K. Sanui and J. Wada, J. Polym. Sci., Polym. Lett. Ed., 1976, $14,459$.

2 A. Harada and M. Kamachi, Macromolecules, 1990, 23, 2821.

3 (a) J. Li and X. J. Loh, Adv. Drug Delivery Rev., 2008, 60, 1000; (b) S. Loethen, J.-M. Kim and D. H. Thompson, Polym. Rev., 2007, 47, 383; (c) T. Ooya and N. Yui, MML Ser., 2006, 7, 231; (d) M. J. Frampton and H. L. Anderson, Angew. Chem., Int. Ed., 2007, 46, 1028; (e) A. Harada, A. Hashidzume, H. Yamaguchi and Y. Takashima, Chem. Rev., 2009, 109, 5974.

4 (a) J. Araki and K. Ito, Soft Matter, 2007, 3, 1456; (b) Y. Okumura and K. Ito, Adv. Mater., 2001, 13, 485.

5 (a) H. Iguchi, S. Uchida, Y. Koyama and T. Takata, ACS Macro Lett., 2013, 2, 527; (b) K. Jang, K. Miura, Y. Koyama and T. Takata, Org. Lett., 2012, 14, 3088; (c) K. Nakazono, T. Takashima, T. Arai, Y. Koyama and T. Takata, Macromolecules, 2010, 43, 691; (d) T. Arai, M. Hayashi, N. Takagi and T. Takata, Macromolecules, 2009, 42, 1881; (e) N. Kihara, K. Hinoue and T. Takata, Macromolecules, 2005, 38, 223; $(f)$ J. Araki, C. Zhao and K. Ito, Macromolecules, 2005, 38, 7524; $(g)$ S. Loethen, T. Ooya, H. S. Choi, N. Yui and D. H. Thompson, Biomacromolecules, 2006, 7, 2501; (h) K. Kato, H. Komatsu and K. Ito, Macromolecules, 2010, 43, 8799.

6 (a) M. E. Belowich, C. Valente, R. A. Smaldone, D. C. Friedman, J. Thiel, L. Cronin and J. F. Stoddart, J. Am. Chem. Soc., 2012, 134, 5243; (b) J. Wu, K. C.-F. Leung and J. F. Stoddart, Proc. Natl. Acad. Sci. U. S. A., 2007, 104, 17266; A. L. Fuller, D. A. Leigh and P. J. Lusby, Angew. Chem., Int. Ed., 2007, 46, 5015; (c) K. M. Wollyung, K. Xu, M. Cochran, A. M. Kasko, W. L. Mattice, C. Wesdemiotis and C. Pugh, Macromolecules, 2005, 38, 2574; $(d)$ N. Watanabe, T. Yagi, N. Kihara and T. Takata, Chem. Commun., 2002, 2720; (e) T.-A. Yamagishi, A. Kawahara, J. Kita, M. Hoshima, A. Umehara and S. Ishida, Macromolecules, 2001, 34, 6565; $(f)$ D. Whang, Y.-M. Jeon, J. Heo and K. Kim, J. Am. Chem. Soc., 1996, 118, 11333. 
7 Polypseudorotaxane (without end-cap group): (a) P. L. Vidal, M. Billon, B. Divisia-Blohorn, G. Bidan, B. Divisia-Blohorn, J. M. Kern and J. P. Sauvage, Chem. Commun., 1998, 629; (b) S. S. Zhu and T. M. Swager, J. Am. Chem. Soc., 1997, 119, 12568; (c) G. J. Owen and P. Hodge, Chem. Commun., 1997, 11; (d) P. E. Mason, I. W. Parsons and M. S. Tolley, Angew. Chem., Int. Ed., 1996, 35, 2238.

8 (a) F. Huang and H. W. Gibson, Prog. Polym. Sci., 2005, 30, 982; (b) F. M. Raymo and J. F. Stoddart, Chem. Rev., 1999, 99, 1643; (c) H. W. Gibson, S. Liu, P. Lecavalier, C. Wu and Y. X. Shen, J. Am. Chem. Soc., 1995, 117, 852; (d) C. Wu, M. C. Bheda, C. Lim, Y. X. Shen, J. Sze and H. W. Gibson, Polym. Commun., 1991, 32, 204.

9 Poly[2]rotaxane synthesis: (a) T. Ikeda, M. Higuchi and D. G. Kurth, J. Am. Chem. Soc., 2009, 131, 9158; (b) D. Tuncel and J. H. G. Steinke, Macromolecules, 2004, 37, 288; (c) T. J. Kidd, T. J. A. loontjens, D. A. Leigh and J. K. Y. Wong, Angew. Chem., Int. Ed., 2003, 42, 3379.

10 Higher order rotaxanated CE-PPRX (without end-caps): (a) D. Loveday, G. L. Wilkes, M. C. Bheda, Y. X. Shen and H. W. Gibson, J. Macromol. Sci., Part A: Pure Appl. Chem., 1995, 32, 1; (b) H. W. Gibson, C. Wu, Y. X. Shen, M. Bheda, J. Sze, P. Engen, A. Prasad, H. Marand, D. Loveday and G. Wilkes, Polym. Prepr., 1991, 32, 637-638. (with end-caps): (c) S.-H. Chiu, S. J. Rowan, S. J. Cantrill, L. Ridvan, P. R. Ashton, R. L. Garrell and J. F. Stoddart, Tetrahedron, 2002, 58, 807.

11 (a) H. Sasabe and T. Takata, J. Porphyrins Phthalocyanines, 2007, 11, 334; (b) Y.-G. Lee, Y. Koyama, M. Yonekawa and T. Takata, Macromolecules, 2010, 43, 4070.
12 T. Ishino, K. Nakazono, Y. Koyama and T. Takata, Polym. Prepr., 2009, 58, 2876.

13 N. Momčilovič, P. G. Clark, A. J. Boydston and R. H. Grubbs, J. Am. Chem. Soc., 2011, 133, 19087.

14 Y. Abe, H. Okamura, S. Uchida and T. Takata, Polym. J., 2014, 46, 553. 15 Y. Furusho, H. Sasabe, D. Natsui, K. Murakawa, T. Takata and T. Harada, Bull. Chem. Soc. Jpn., 2004, 77, 179.

16 T. Perner and R. C. Schultz, Br. Polym. J., 1987, 19, 181.

$17 K_{\mathrm{a}}\left[\mathrm{Bn}_{2} \mathrm{NH}_{2} \cdot \mathrm{PF}_{6}-\mathrm{DB} 24 \mathrm{C} 8\right]=420 \mathrm{M}^{-1}, K_{\mathrm{a}}\left[n-\mathrm{Bu}_{2} \mathrm{NH}_{2} \cdot \mathrm{PF}_{6}-\mathrm{DB} 24 \mathrm{C} 8\right]=$ $50 \mathrm{M}^{-1}$ (298 K, $\left.\mathrm{CD}_{3} \mathrm{CN}\right)$ : P. R. Ashton, P. J. Campbell, E. J. T. Chrystal, P. T. Glink, S. Menzer, D. Philp, N. Spencer, J. F. Stoddart, P. A. Tasker and D. J. Williams, Angew. Chem., 1995, 107, 1997 (Angew. Chem., Int. Ed., 1995, 34, 1865).

18 The CE is perhaps likely to translate to the inner ammonium site on the axle rather than dethreading to gain in enthalpy.

19 (a) Y. Tachibana, H. Kawasaki, N. Kihara and T. Takata, J. Org. Chem., 2006, 71, 5093; (b) N. Kihara, Y. Tachibana, H. Kawasaki and T. Takata, Chem. Lett., 2000, 506.

20 H. Kawasaki, N. Kihara and T. Takata, Chem. Lett., 1999, 1015.

21 Measurable dissociation of polypseudorotaxne was confirmed in DMSO at room temperature.

22 See ESI $\dagger$.

23 Wheel components move in each phase between acetyl-acetyl groups that are not sequential on the axle polymer: N. Kihara, Y. Koike and T. Takata, Chem. Lett., 2007, 36, 208. 